\title{
Is fecal microbiota transplantation a promising strategy for type 2 diabetes mellitus?
}

\section{Opinion}

The gut microbiota is an ecosystem consisted of an estimated 10100 trillion microorganisms, including bacteria, fungi, archaea, and viruses, all of which colonize in the human gastrointestinal tract. ${ }^{1}$ Gut microbiota exerts diverse physiological functions, such as modulation of immune system, regulation of the gut barrier integrity and biosynthesis of vitamins. Imbalance in gut microbial composition and function, commonly known as dysbiosis, has been closely associated with a variety of diseases, including Clostridium difficile infection (CDI), obesity, cancer and type 2 diabetes mellitus (T2DM).,

As one of the global top health issues, T2DM is believed to be caused by various factors, such as gene alterations, sedentary lifestyle, poor diet and obesity. ${ }^{4}$ The precise mechanism of T2DM is not completely clear, accumulating evidences have suggested gut microbiota is tightly involved in the development of the disease. Two large metagenome-wide association studies in China and Europe revealed that individuals with T2DM were characterized by gut microbial dysbiosis, with a decrease in the abundance of some universal butyrate-producing bacteria (Roseburia), and an increase in various opportunistic pathogens (Clostridium clostridioforme). ${ }^{5,6}$ Other studies also reported significant compositional and functional alterations of microbiota in T2DM patients, compared to healthy people. ${ }^{7-9}$ The role of altered gut microbiota may not only be the consequences of T2DM, but also the causalities in developing T2DM. ${ }^{10}$ The mechanism might be related to the increase of gut permeability, microbial translocation and lipopolysaccharides (LPS), as well as the decrease of short-chain fatty acids (SCFAs), alterations of intestinal immune system, bile acids (BAs), branched chain amino acids, trimethylamine N-oxide (TMAO) and endocannabinoid system. ${ }^{11-15}$

Fecal microbiota transplantation (FMT) is a promising strategy to alter microbiota composition and function, and also to treat microorganisms related diseases. It is well known as to transfer the fecal materials from a healthy donor to a recipient, in order to alter the microbiome inside the recipient. FMT could enhance gut microbiota diversity, resulting in disease regression in $92 \%$ patients with CDI, which is a gastrointestinal disease believed to be caused by perturbations in the intestinal microbiota. ${ }^{16}$ Regarding recurrent $\mathrm{CDI}$, guidelines recommended FMT as the standard treatment. ${ }^{17,18} \mathrm{As}$ for T2DM, limited evidences have reported the relationship between FMT and diabetic patients. However, multiple FMT clinical trials in T2DM are ongoing (NCT03127696, NCT02346669, NCT01790711 and NCT01790711). The benefial effects of FMT for individuals with T2DM may be hypothesized from the results of patients with metabolic syndromes. Vrieze et al reported the insulin sensitivity of recipients with metabolic syndromes was upregulated, along with the levels of butyrate-producing intestinal microbiota, six weeks later after transfering microbiota obtained from lean donors. ${ }^{19}$ Another study also reported that in individuals with metabolic syndromes, glycated hemoglobin (HbAlc) was significantly reduced after allogenic FMT, and this phenomenon was associated with changes in intestinal microbiota. ${ }^{20}$ Moreover, a reduced gene richness of baseline microorganism before lean donor FMT was related with a better
Volume 8 Issue 2 - 2020

\author{
Zhongping Yang \\ Chongqing Public Health Medical Center, China
}

Correspondence: Zhongping Yang, Chongqing Public Health Medical Center, 109 Baoyu Road, Shapingba District, Chongqing, China, Email I04658027@qq.com

Received: December 04, 2019 | Published: March 17, 2020

clinical outcome. ${ }^{20}$ Therefore, modulation of gut microbiota with FMT might be an extremely promising treatment option for T2DM.

Even so, some significant points about FMT for diabetic patients remain to be illustrated. To begin with, since most studies are still ongoing, the efficacy of FMT are still unclear. Then, we also need to identify the specific type of patiets, who might benefit the most from FMT. Thirdly, influence factors should be evaluated, such as FMT dose, administration route, working duration, and donors' microbiota profile. Lastly, as for some reported cases of intestinal bleeding and peritonitis during nasogastric tube administration of FMT, ${ }^{21}$ there are also concerns about transmission of communicable diseases and increased infection risk with regard to FMT. Thus, there is still a long way to go before FMT could be applied in diabetic patients.

In conclusion, growing evidences indicate that gut microbiota plays an indispensable role in T2DM progress via multiple mechanisms. As a prospective strategy to modulate microbiota composition and function, FMT has been proved to be effective in CDI and other diseases. Despite existence of multiple challenges and obstacles, appropriately administered FMT is believed to be a boon for individuals with T2DM.

\section{Acknowledgments}

None.

\section{Conflicts of interest}

The author declares that there are no conflicts of interest.

\section{Funding}

None.

\section{References}

1. Integrative HMPRNC. The Integrative Human Microbiome Project. Nature. 2019;569(7758):641-648.

2. Reimer RA. Establishing the role of diet in the microbiota-disease axis. Nat Rev Gastroenterol Hepatol. 2019;16(2):86-87. 
3. Sterlin D. The antibody/microbiota interface in health and disease Mucosal Immunol. 2019.

4. Salamon D. Characteristics of gut microbiota in adult patients with type 1 and type 2 diabetes based on nextgeneration sequencing of the $16 \mathrm{~S}$ rRNA gene fragment. Pol Arch Intern Med. 2018;128(6):336-343.

5. Karlsson FH. Gut metagenome in European women with normal, impaired and diabetic glucose control. Nature. 2013;498(7452):99-103.

6. Qin J. A metagenome-wide association study of gut microbiota in type 2 diabetes. Nature. 2012;490(7418):55-60.

7. Jamshidi P. Is there any association between gut microbiota and type 1 diabetes? A systematic review. Gut Pathog. 2019;11:49.

8. Moon JY. Gut microbiota and plasma metabolites associated with diabetes in women with, or at high risk for, HIV infection. EBioMedicine. 2018;37:392-400.

9. Maffeis C. Association between intestinal permeability and faeca microbiota composition in Italian children with beta cell autoimmunity at risk for type 1 diabetes. Diabetes Metab Res Rev. 2016;32(7):700-709.

10. Meijnikman AS. Evaluating Causality of Gut Microbiota in Obesity and Diabetes in Humans. Endocr Rev. 2018;39(2):133-153.

11. Zhang B. Gut Microbiota, a Potential New Target for Chinese Herbal Medicines in Treating Diabetes Mellitus. Evid Based Complement Alternat Med. 2019;2019:1-12.

12. Sircana A. Altered Gut Microbiota in Type 2 Diabetes: Just a Coincidence? Curr Diab Rep. 2018;18(10):98.

13. Bloomgarden Z. Diabetes and branched-chain amino acids: What is the link? J Diabetes. 2018;10(5):350-352.
14. Shan Z. Association between microbiota-dependent metabolite trimethylamine-N-oxide and type 2 diabetes. Am J Clin Nutr. 2017;106(3):888-894.

15. Cani PD. Endocannabinoids - at the crossroads between the gut microbiota and host metabolism. Nat Rev Endocrinol. 2016;12(3):133-143.

16. Gough E, Shaikh H, Manges AR. Systematic review of intestinal microbiota transplantation (fecal bacteriotherapy) for recurrent Clostridium difficile infection. Clin Infect Dis. 2011;53(10):994-1002.

17. McDonald LC. Clinical Practice Guidelines for Clostridium difficile Infection in Adults and Children: 2017 Update by the Infectious Diseases Society of America (IDSA) and Society for Healthcare Epidemiology of America (SHEA). Clin Infect Dis. 2018;66(7):e1-e48.

18. Surawicz CM. Guidelines for diagnosis, treatment, and prevention of Clostridium difficile infections. Am J Gastroenterol. 2013;108(4):478-498.

19. Vrieze A. Transfer of intestinal microbiota from lean donors increases insulin sensitivity in individuals with metabolic syndrome. Gastroenterology. 2012;143(4):913-917.

20. Kootte RS. Improvement of Insulin Sensitivity after Lean Donor Feces in Metabolic Syndrome Is Driven by Baseline Intestinal Microbiota Composition. Cell Metab. 2017;26(4):611-619.

21. Kassam Z. Fecal microbiota transplantation for Clostridium difficile infection: systematic review and meta-analysis. Am J Gastroenterol. 2013;108(4):500-508 